(C) 1981. The Genetical Society of Great Britain

\title{
A GENERAL ANALYSIS OF GENETIC MODELS WITH FREQUENCY-DEPENDENT MATING. II. SEXUAL SELECTION FOR HETEROZYGOTES
}

\author{
I. St. LAWRENCE and P. O'DONALD
}

Department of Genetics, University of Cambridge, Downing Street, Cambridge CB2 3EH

Received 23.x.80

\section{SUMMARY}

\begin{abstract}
Heterozygotes are assumed to mate with a frequency that is any general function, $f(v)$, of their population frequency, $v$. Models are analysed in which the selection that determines the function $f(v)$ acts either on one sex alone or on both sexes equally. The central equilibrium point $v_{*}=\frac{1}{2}$ always exists; it is stable if $f\left(\frac{1}{2}\right)>\frac{1}{2}$. If the central equilibrium is unstable, other asymmetric equilibria may be stable; the fixation states may also be stable. This general analysis is applied to a number of specific models of sexual selection. The models give qualitatively different results. The outcome of selection in population cage experiments could be used to test the alternative models.
\end{abstract}

\section{INTRODUCTION}

IN the first paper of this series, O'Donald $(1980 \mathrm{~b})$ analysed models of selection in which the rates of mating were general functions of the frequencies of the phenotypes. Thus, with two phenotypes $A$ and $B$, the probabilities of mating are given by

$$
\begin{aligned}
& P_{T}(A)=g(w) \\
& P_{T}(B)=1-g(w)
\end{aligned}
$$

where $g(w)$ is any function of the frequency $w$ of the $B$ phenotype. This model subsumes a wide range of models of sexual and natural selection. For example, in the simplest model of sexual selection, a proportion $\alpha$ of the females prefer to mate with $A$ males and a proportion $\beta$ prefer to mate with $B$ males. Then the males mate with probabilities

$$
\begin{aligned}
& P_{T}(A)=\alpha+(1-w)(1-\alpha-\beta)=g(w) \\
& P_{T}(B)=1-g(w)=\beta+w(1-\alpha-\beta) .
\end{aligned}
$$

Models of frequency-dependent natural selection (e.g., Clarke and O'Donald, 1964) can also be described in these terms.

In the models previously analysed (Karlin, 1978; O'Donald, 1980 b) $A$ was assumed to be dominant to $B$. Simple, general formulae were then obtained for the equilibrium frequency and the eigenvalue giving the rate of approach to equilibrium. In the genetical formulation of the model, there are at least three different genotypes:

$A A$ and $A a$ representing the phenotype $A$; $a a$ representing the phenotype $B$.

The frequencies of these genotypes can be represented by the vector $(u$, $v, w)$ and since $u+v+w=1$ only two frequencies are independent. These 
genetical models can be described most conveniently by the pair of frequencies $p$ and $w$ where $p$ is the gene frequency of the allele $A$, given by

$$
p=u+\frac{1}{2} v \text {. }
$$

The matings were assumed to take place at random between the phenotypes. This entails that, at equilibrium, Hardy-Weinberg ratios are maintained. All sexual selection models in which the phenotypes or genotypes do not assort show this feature (O'Donald, 1980a). On the way to equilibrium, however, the genotypic frequencies do show deviations from the Hardy-Weinberg values. Selection was assumed to act either on males alone or on both sexes. A character for sexual display, sex-limited to males, will give rise to frequencies of matings, $g(w)$ for $A$ and $1-g(w)$ for $B$, that apply only to the males: the females, on the other hand, mate with population frequencies, $1-w$ for $A$ and $w$ for $B$. If selection acts equally on both sexes-an assumption valid often for natural selection but seldom for sexual selection-then both sexes mate with phenotypic frequencies $g(w)$ and $1-g(w)$.

For the model with dominant and recessive phenotypes, the equilibrium frequency of the recessive $B$ is always given by a solution of the equation

$$
w_{*}=1-g\left(w_{*}\right) \text {. }
$$

$w_{*}$ is the equilibrium frequency both when selection acts only on males and when selection acts on both sexes. The rate of approach to this equilibrium is the same for both the $p$ and $w$ dimension. It is given by the leading eigenvalue of the gradient matrix. This can be found in the following way. Recursion equations are obtained for the frequencies of the allele $A$ and the genotype $a a$. These equations express the frequencies in generation $t+1$ as functions of the frequencies in generation $t$. If $p^{\prime}$ and $w^{\prime}$ are the frequencies in $t+1$ and $p$ and $w$ the frequencies in $t$, then the gradient matrix is defined as

$$
\left(\begin{array}{ll}
\frac{\partial p^{\prime}}{\partial p_{*}} & \frac{\partial p^{\prime}}{\partial w_{*}} \\
\frac{\partial w^{\prime}}{\partial p_{*}} & \frac{\partial w^{\prime}}{\partial w_{*}}
\end{array}\right)
$$

where the symbols $\partial p^{\prime} / \partial p_{*}, \partial p^{\prime} / \partial w_{*}$ are used to indicate that the partial derivatives are to be evaluated at the equilibrium points $p=p_{*}$ and $w=w_{*}$. Since the Hardy-Weinberg ratios are reached at equilibrium

$$
\begin{aligned}
& q_{*}=\sqrt{w_{*}} \\
& p_{*}=1-\sqrt{w_{*}} .
\end{aligned}
$$

For the general model of selection of males with dominant and recessive phenotypes, the gradient matrix takes the form

$$
\left(\begin{array}{cc}
1 & \frac{\frac{1}{2} \theta}{q_{*}} \\
-2 q_{*} & -\theta
\end{array}\right)
$$


giving the two eigenvalues, one zero and the other $\lambda=1-\theta$, where

$$
\theta=\frac{p_{*} q_{*}\left(1+d g / d w_{*}\right)}{g\left(w_{*}\right)} .
$$

When selection acts on both sexes, the non-zero eigenvalue is simply

$$
\lambda=1-2 \theta \text {. }
$$

Since there is only one eigenvalue, there is only one direction of approach to the equilibrium. Near equilibrium $\lambda$ denotes the values of the deviations from the equilibrium frequencies in successive generations, and

$$
\frac{p^{\prime}-p_{*}}{p-p_{*}}=\frac{w^{\prime}-w_{*}}{w-w_{*}}=\lambda \text {. }
$$

Provided $\lambda<1, p^{\prime}$ and $w^{\prime}$ converge to $p_{*}$ and $w_{*}$. The condition $\lambda<1$ thus determines the "local" stability of the equilibrium - the stability within a region close to equilibrium where the linear approximation is valid. Global stability can often be established for specific models of the function $g(w)$, by direct manipulation of the system of recursion equations.

O'Donald (1980a) analysed specific models of selection in which the heterozygotes determine one of the phenotypes and the two homozygotes determine the other phenotype. This genetical model can give rise to a multiplicity of equilibria in special cases. In this paper we analyse general selection models with heterozygous snd homozygous phenotypes. The multiplicity of equilibria found in some special cases is shown to be a general phenomenon. The conditions are given for the stability of the alternative equilibria. The results are then applied to particular models and the evolutionary consequences of selection analysed.

\section{Selection of males alone}

The genotypes $A A, A a$ and $a a$ occur with population frequencies $u$, $v$ and $w$. They determine two phenotypes: a phenotype which is genotypically $A a$ and a phenotype which is genotypically either $A \boldsymbol{A}$ or $a a$. In terms of sexual selection, there might be mating preferences among the females either for $A a$ or for $A A$ and $a a$. Then the males' probabilities of mating can be described by the function $f$ of the heterozygote frequency $v$. Thus,

$$
\begin{aligned}
P_{T}(A a) & =f(v) \\
P_{T}(A A \text { or } a a) & =1-f(v),
\end{aligned}
$$

Individual males and females thus mate with genotypic frequencies as

$\begin{array}{ccc}\text { Genotypes } & \text { Males } & \text { Females } \\ A A & \frac{u(1-f)}{(1-v)} & u \\ A a & f & v \\ a a & \frac{w(1-f)}{(1-v)} & w\end{array}$


This then gives rise to mating frequencies

Matings Frequencies

$$
\begin{array}{cc}
A A \times A A & \frac{u^{2}(1-f)}{(1-v)} \\
A A \times A a & \frac{u v(1-f)}{(1-v)+u f} \\
A A \times a a & \frac{2 u w(1-f)}{(1-v)} \\
A a \times A a & \frac{v w(1-f)}{(1-v)}+w f \\
A a \times a a & \frac{w^{2}(1-f)}{(1-v)} \\
a a \times a a &
\end{array}
$$

Hence we obtain the recursion equations for the genotypic frequencies:

$$
\begin{aligned}
& u^{\prime}=\frac{u p(1-f)}{(1-v)}+\frac{1}{2} p f \\
& v^{\prime}=\frac{2 u w(1-f)}{(1-v)}+\frac{1}{2} f+\frac{1}{2} v(1-f) \\
& w^{\prime}=\frac{w q(1-f)}{(1-v)}+\frac{1}{2} q f .
\end{aligned}
$$

Here $p$ and $q$ are the allelic frequencies of $A$ and $a$. Since in the next generation $A$ takes the frequency

$$
p^{\prime}=u^{\prime}+\frac{1}{2} v^{\prime}
$$

we obtain a difference equation in the gene frequency

$$
\begin{aligned}
\Delta p & =p^{\prime}-p \\
& =\frac{\frac{1}{2}\left(p-\frac{1}{2}\right)(v-f)}{(1-v)} .
\end{aligned}
$$

At equilibrium $\Delta p=0$ and either

$$
p_{*}=\frac{1}{2}
$$

or

$$
v_{*}=f\left(v_{*}\right)
$$

define the equilibrium frequencies. There are at least two alternative equilibria, and more than two if the equation

$$
v_{*}=f\left(v_{*}\right)
$$


has more than a single root. It is easily shown that at all equilibria, the Hardy-Weinberg Law holds. For example, when $f=v$

$$
u^{\prime}=u p+\frac{1}{2} p v=p^{2}
$$

hence

$$
w^{\prime}=q^{2}
$$

and

$$
v^{\prime}=2 p q .
$$

To determine the local stability of these equilibria, we consider the pair of equations

$$
\begin{aligned}
& p^{\prime}=\frac{2 p(2-v-f)+(f-v)}{4(1-v)} \\
& v^{\prime}=\frac{1}{2} f+\frac{1}{2} v(1-f)+\frac{\left(2 p q-v+\frac{1}{2} v^{2}\right)(1-f)}{(1-v)} .
\end{aligned}
$$

At the equilibrium $v_{*}=f\left(v_{*}\right)$, we obtain the gradient matrix

$$
\left(\begin{array}{cc}
1 & \frac{(q-p)}{4(1-v)}\left(\frac{d f}{d v}-1\right) \\
2(q-p) & \frac{(q-p)^{2}}{2(1-v)}\left(\frac{d f}{d v}-1\right)
\end{array}\right)
$$

This matrix has the non-zero eigenvalue

$$
\lambda=1+\frac{(q-p)^{2}}{2(1-v)}\left(\frac{d f}{d v}-1\right) .
$$

Hence the condition for stability of the equilibrium $v_{*}=f\left(v_{*}\right)$ becomes

$$
\lambda<1
$$

or

$$
\frac{d f}{d v}<1
$$

At the equilibrium $p_{*}=\frac{1}{2}$ the gradient matrix is simply

$$
\left(\begin{array}{cc}
\frac{3}{2}-f & 0 \\
0 & 0
\end{array}\right)
$$

and

$$
\lambda=\frac{3}{2}-f \text {. }
$$

Since the Hardy-Weinberg Law holds at this equilibrium we have the genotypic frequencies

$$
\begin{gathered}
u_{*}=w_{*}=\frac{1}{4} \\
v_{*}=\frac{1}{2} .
\end{gathered}
$$


The condition for stability is $\lambda<1$ or

$$
f>\frac{1}{2}
$$

i.e., $f\left(\frac{1}{2}\right)>\frac{1}{2}$. This implies that the heterozygous males must have a mating advantage over the homozygotes: heterozygous advantage must prevail, otherwise this equilibrium will be unstable. If it is unstable, then the equation

$$
v_{*}=f\left(v_{*}\right)
$$

will either define a stable equilibrium point, or the fixation states $(1,0,0)$ and $(0,0,1)$ will be stable and no polymorphism can be maintained. The equation may often have, as one of its roots, the solution

$$
v_{*}=0 \text {. }
$$

This solution of course entails fixation at either $(1,0,0)$ or $(0,0,1)$

\section{SELECTION OF BOTH SEXES EQUALLy}

Analysis of this model proceeds in a similar way to that for selection of males alone. The matings now take place at frequencies

$$
\begin{array}{ll}
\text { Matings } & \text { Frequencies } \\
A A \times A A & {\left[\frac{u(1-f)}{(1-v)}\right]^{2}} \\
A A \times A a & \frac{2 u f(1-f)}{(1-v)} \\
A A \times a a & 2 u w\left[\frac{(1-f)}{(1-v)}\right]^{2} \\
A a \times A a & \frac{2 w f(1-f)}{(1-v)} \\
A a \times a a & {\left[\frac{w(1-f)}{(1-v)}\right]^{2}} \\
a a \times a a &
\end{array}
$$

From the genotypic recursion equations

$$
\begin{gathered}
u^{\prime}=\left[\frac{u(1-f)}{(1-v)}+\frac{1}{2} f\right]^{2} \\
v^{\prime}=f(1-f)+2 u w\left[\frac{(1-f)}{(1-v)}\right]^{2}+\frac{1}{2} f^{2} \\
w^{\prime}=\left[\frac{w(1-f)}{(1-v)}+\frac{1}{2} f\right]^{2}
\end{gathered}
$$

we obtain the difference equation of gene frequency

$$
\Delta p=\frac{\left(p-\frac{1}{2}\right)(v-f)}{(1-v)}
$$


which is exactly twice the difference when selection acts on males alone. The gradient matrix is then

$$
\left(\begin{array}{cc}
1 & \frac{(1-v)}{2(1-v)}\left(\frac{d f}{d v}-1\right) \\
2(q-p) & \frac{(q-p)^{2}}{(1-v)}\left(\frac{d f}{d v}-1\right)
\end{array}\right)
$$

giving the non-zero eigenvalue

$$
\lambda=1-2 \theta
$$

where

$$
\theta=\frac{(q-p)^{2}}{(1-v)}\left(1-\frac{d f}{d v}\right)
$$

compared to the eigenvalue

$$
\lambda=1-\theta
$$

for selection of males alone. In both cases of selection the stability condition $\lambda<1$ entails

$$
\frac{d f}{d v}<1
$$

The central equilibrium $p_{*}=1 / 2$ is approached at a rate given by

$$
\lambda=2(1-f)
$$

for

$$
\lambda<1
$$

we must have

$$
1-f<\frac{1}{2}
$$

or

$$
f\left(\frac{1}{2}\right)>\frac{1}{2}
$$

giving the same condition of heterozygous advantage as when selection acts on males alone.

The possibility also arises that both sexes may be selected, but one sex selected differently from the other. This would happen for example if both sexes were subjected to natural selection but only the males were subjected to sexual selection. In this case, the gradient matrix has no simple general form and specific models are best analysed separately.

\section{Applications of the general formulaE to SPECific models}

In this section the general formulae will be applied to specific models. The rates of approach to equilibrium will also be analysed numerically. The following specific models are analysed: sexual selection with fixed mating preferences; encounter models of sexual selection (O'Donald, 1978, $1980 a)$; sexual selection for the heterozygotes and natural selection against 
them; sexual selection for the homozygotes and natural selection against them.

\section{(i) Fixed preference model of sexual selection}

In this model, a proportion $\alpha$ of the females prefer homozygous males and $\beta$ prefer heterozygous males. Then we have

$$
P_{T}(A a)=f(v)=\beta+v(1-\alpha-\beta) .
$$

The equilibrium $v_{*}=\frac{1}{2}\left(p_{*}=\frac{1}{2}\right)$ will be stable if $f\left(\frac{1}{2}\right)>\frac{1}{2}$ which is equivalent to

$$
\beta>\alpha \text {. }
$$

More females must prefer heterozygotes than prefer homozygotes.

The equilibrium $v_{*}=f\left(v_{*}\right)$ has the solution

$$
v_{*}=\frac{\beta}{(\alpha+\beta)}
$$

and will be stable provided that

$$
\alpha>\beta \text {. }
$$

This follows because

$$
\frac{d f}{d v}-1=-\alpha-\beta<0
$$

for all values of $\alpha$ and $\beta$. But since we must have

$$
v_{*} \leqq \frac{1}{2}
$$

\begin{tabular}{|c|c|c|c|}
\hline Equilibrium & Conditions & atisfied & \\
\hline frequency & $\beta>\alpha$ & $\alpha>\beta$ & Eigenvalue \\
\hline$v_{*}=\frac{1}{2}$ & stable & unstable & $\lambda=1-\frac{1}{2}(\beta-\alpha)$ \\
\hline$v_{*}=\frac{\beta}{(\alpha+\beta)}$ & unstable & stable & $\lambda=1-\frac{1}{2}\left(\alpha^{2}-\beta^{2}\right) / \alpha$ \\
\hline
\end{tabular}

given Hardy-Weinberg frequencies at equilibrium, therefore

$$
\frac{\beta}{(\alpha+\beta)}<\frac{1}{2}
$$

giving the condition $\beta<\alpha$ as above. These results may be summarized as follows:

(ii) Encounter model of sexual selection

This model is more realistic than the model with fixed preference. A female mates preferentially if she encounters a male she prefers within a certain number of random encounters with courting males (O'Donald, $1978,1979,1980 a$ ); after these encounters, she mates with the next mate she meets regardless of his type 
The overall probabilities of mating are as follows:

$$
\begin{gathered}
P_{T}(A A)+P_{T}(a a)=\alpha\left(1-v^{n+1}\right)+\beta(1-v)^{m+1}+(1-v)(1-\alpha-\beta) \\
P_{T}(A a)=f(v)=\alpha v^{n+1}+\beta\left[1-(1-v)^{m+1}\right]+v(1-\alpha-\beta) .
\end{gathered}
$$

Two cases of the encounter model give rise to simple analytical results for the model with heterozygous and homozygous phenotypes: Case (i), $m=$ $n=1$; Case (ii) $m=n=2$.

Case $(i): m=n=1$. For this case we have

$$
f(v)=(\alpha-\beta) v^{2}+v(1-\alpha+\beta)
$$

giving rise to theoretical equilibria

$$
v_{*}=\frac{1}{2}, 0,1 \text {. }
$$

The equilibrium $v_{*}=1$ is obviously impossible. $v_{*}=0$ implies that one or other homozygote must become fixed. The difference equation in gene frequency

$$
\begin{aligned}
\Delta p & =\frac{\frac{1}{2}\left(p-\frac{1}{2}\right)(v-f)}{(1-v)} \\
& =\frac{1}{2} v\left(p-\frac{1}{2}\right)(\alpha-\beta) .
\end{aligned}
$$

If $\alpha>\beta$, then for all $p>\frac{1}{2}, \Delta p>0$ defines the domain of attraction to $(1,0,0)$. For all $p<\frac{1}{2}, \Delta p<0$ defines the domain attraction to $(0,0,1)$. If $\beta>\alpha$, then for all $p>\frac{1}{2}, \Delta p<0$ and for all $p<\frac{1}{2}, \Delta p>0$ showing that there is global convergence to

$$
p_{*}=v_{*}=\frac{1}{2} \text {. }
$$

Heterozygosity in mating preference is thus required for the maintenance of any polymorphism. The eigenvalue of the rate of approach to $p_{*}=\frac{1}{2}$ is given by

$$
\lambda=1-\frac{1}{4}(\beta-\alpha) .
$$

Case (ii): $m=n=2$. Now we have

$$
f(v)=\alpha v^{3}+\beta\left[1-(1-v)^{3}\right]+v(1-\alpha-\beta) .
$$

This leads to the gene frequency difference equation

$$
\Delta p=\frac{1}{2} v\left(p-\frac{1}{2}\right)[(\alpha+\beta) v+\alpha-2 \beta] .
$$

The condition $\alpha>2 \beta$ ensures that $\Delta p>0$ for $p>\frac{1}{2}$ and $\Delta p<0$ for $p<\frac{1}{2}$, thus giving the domains of attraction to the two fixation states $(1,0,0)$ and $(0,0,1)$.

Two stable equilibria can exist if $\alpha<2 \beta$. The condition $\alpha<\beta$ ensures that since $v \leqq \frac{1}{2}$ therefore

$$
(\alpha+\beta) v-2 \beta+\alpha<0 .
$$

Thus there is global convergence to $p_{*}=\frac{1}{2}$, at a rate given by the eigenvalue

$$
\lambda=1-\frac{3}{8}(\beta-\alpha) \text {. }
$$


The condition $2 \beta>\alpha>\beta$ gives rise to stability of the equilibrium

$$
v_{*}=\frac{(2 \beta-\alpha)}{(\alpha+\beta)} .
$$

The eigenvalue is given by

$$
\begin{aligned}
\lambda & =1+\frac{(q-p)^{2}}{2(1-v)}\left(\frac{d f}{d v}-1\right) \\
& =1+\frac{3(2 \beta-\alpha)(\beta-\alpha)}{2(\alpha+\beta)}
\end{aligned}
$$

For stability $\lambda<1$ which requires that

$$
(2 \beta-\alpha)(\beta-\alpha)<0
$$

or

$$
2 \beta>\alpha>\beta .
$$

The results for this case can be summarized as follows:

$$
\begin{aligned}
& \text { Equilibrium Conditions satisfied } \\
& \text { frequency } \quad \alpha<\beta \quad \beta<\alpha<2 \beta \quad 2 \beta<\alpha \quad \text { Eigenvalue } \\
& v_{*}=\frac{1}{2} \quad \text { stable unstable unstable } \quad \lambda=1-\frac{3}{8}(\beta-\alpha) \\
& v_{*}=\frac{(2 \beta-\alpha)}{\alpha+\beta)} \quad \text { unstable stable unstable } \lambda=1+\frac{3(2 \beta-\alpha)(\beta-\alpha)}{2(\alpha+\beta)} \\
& v_{*}=0 \text { (fixation unstable unstable stable } \\
& \text { states) }
\end{aligned}
$$

(iii) Sexual and natural selection of heterozygotes

In this model, the heterozygotes are assumed to be the preferred phenotypes, which have a lowered viability compared to the non-preferred homozyotes. We let the selective coefficient of the heterozygote be $t$. Then, after natural selection, heterozygotes and homozygotes occur with frequencies

$$
\begin{aligned}
A a & \frac{v(1-t)}{(1-t v)} \\
A A, a a & \frac{(1-v)}{(1-t v)}
\end{aligned}
$$

so that male heterozygotes mate with frequency

$$
P_{T}(A a)=f(v)=\beta+\frac{v(1-\beta)(1-t)}{(1-t v)} .
$$

This gives

$$
\Delta p=\frac{\frac{1}{2}\left(p-\frac{1}{2}\right)(t v-\beta)}{(1-t v)} .
$$


If $2 \beta>t$, this ensures that $t v-\beta<0$. Therefore, if $p>\frac{1}{2}, \Delta p<0$ and if $p<{ }_{2}, \Delta p>0, p_{*}=\frac{1}{2}$ is a globally stable equilibrium. The equilibrium

$$
v_{*}=\beta / t
$$

implies that $\beta / t<\frac{1}{2}$ or $2 \beta<t$. The eigenvalue

$$
\lambda=1+\frac{\frac{1}{2}(2 \beta-t)}{(1-\beta)}
$$

shows that the equilibrium $v_{*}=\beta / t$ is stable, since the condition $2 \beta<t$

\begin{tabular}{|c|c|c|c|}
\hline Equilibrium & Conditions & tisfied & \\
\hline frequency & $2 \beta>t$ & $2 \beta<t$ & Eigenvalues \\
\hline$v_{*}=\frac{1}{2}$ & stable & unstable & $\lambda-1+\frac{\frac{1}{2}(t-2 \beta)}{(2-t)}$ \\
\hline$v_{*}=\frac{\beta}{t}$ & unstable & stable & $\lambda=1+\frac{\frac{1}{2}(2 \beta-t)}{(1-\beta)}$ \\
\hline
\end{tabular}
entails $\lambda<1$. These results can thus be summarized:

(iv) Sexual and natural selection of homozygotes

The homozygotes are now assumed to be the preferred phenotypes, with a selective disadvantage, $s$, in viability. Then heterozygous males mate with frequency

$$
f(v)=\frac{v(1-\alpha)}{1-s(1-v)}
$$

This gives the difference equation

$$
\Delta p=\frac{\frac{1}{2} v\left(p-\frac{1}{2}\right)(\alpha-s+s v)}{(1-v)}
$$

Unlike the case of sexual and natural selection of heterozygotes, the fixation states also exist and are stable under certain conditions. Clearly, if $v_{*}=0$ and $s>\alpha$, then $\Delta p>0$ if $p>\frac{1}{2}$ and $\Delta p<0$ if $p<\frac{1}{2}$. These conditions define the domains of convergence to the fixation states $(1,0,0)$ and $(0,0,1)$.

The condition $s>2 \alpha$ defines global convergence to $p_{*}=\frac{1}{2}$. The other polymorphic equilibrium

$$
v_{*}=1-\frac{\alpha}{s}
$$

has eigenvalue

$$
\lambda=1+\frac{(2 \alpha-s)(\alpha-s)}{2 \alpha(1-\alpha)^{2}}
$$

Thus for stability, $(2 \alpha-s)(\alpha-s)<0$ or

$$
\alpha<s<2 \alpha \text {. }
$$


Summarizing these results:

$$
\begin{aligned}
& \text { Equilibrium Conditions satisfied } \\
& \text { frequency } s<\alpha \quad \alpha<s<2 \alpha \quad 2 \alpha<s \quad \text { Eigenvalues } \\
& v_{*}=\frac{1}{2} \quad \text { unstable unstable stable } \quad \lambda=1+\frac{\frac{1}{2}(2 \alpha-s)}{(2-s)} \\
& v_{*}=1-\frac{\alpha}{s} \quad \text { unstable stable unstable } \lambda=1+\frac{(2 \alpha-s)(\alpha-s)}{2 \alpha(1-\alpha)^{2}} \\
& v_{*}=0 \text { (fixation stable unstable unstable } \\
& \text { states) }
\end{aligned}
$$

\section{NUMERICAL ANALYSIS OF SPECIFIC MODELS}

Each model was analysed numerically by computing the genotypic frequencies in successive generations. Hardy-Weinberg ratios are closely approached in the first, second or third generation. Thereafter the genotypic frequencies lie on the "Hardy-Weinberg line" given by the equation

or

$$
2 p q=v
$$

$$
p=\frac{1}{2} \pm \frac{1}{2} \sqrt{(1-2 v)} \text {. }
$$

The same eigenvalue determines the rate of approach of both $p$ and $v$ along this line to their equilibrium frequencies.

During the approach to equilibrium, the rate of convergence is very close to the geometric rate given by the leading eigenvalue of the gradient matrix at the equilibrium point. Consider, for example, the encounter model of the expression of preference with parameters $m=n=2$. When $\alpha<\beta$ a stable equilibrium is reached at

$$
p_{*}=v_{*}=\frac{1}{2} \text {. }
$$

The eigenvalue as given in section 4 (ii) is

$$
\lambda=1-3(\beta-\alpha) / 8 \text {. }
$$

For example, if $\alpha=0.4$ and $\beta=0.5$, then

$$
\lambda=0.9625 \text {. }
$$

This value can be compared with the actual rate at which equilibrium is approached in successive generations: the ratios of the deviations from equilibrium in one generation and the next should approximately equal the value

$$
\lambda=0.9625 \text {. }
$$

For the generations 96 to 100 we obtain values as follows.

$\begin{array}{ccccc}\text { Generation } & v & \lambda & p & \lambda \\ 96 & 0.4999875 & 0.92644 & 0.5025039 & 0.96250 \\ 97 & 0.4999884 & 0.92637 & 0.5024100 & 0.96250 \\ 98 & 0.4999893 & 0.92648 & 0.5023196 & 0.96250 \\ 99 & 0.4999900 & 0.92637 & 0.5022326 & 0.96250\end{array}$


The rate of convergence of $p$ to its equilibrium is much closer to the theoretical rate than the rate of convergence of $v$. Fluctuations in the rate, admittedly very small, occur from one generation to the next, but these may well be a consequence of rounding errors in the computation.

The statistical behaviour of the other models is essentially similar to that of the encounter model: after a few generations the frequencies follow the Hardy-Weinberg line to equilibrium. Figure 1 shows the rate of

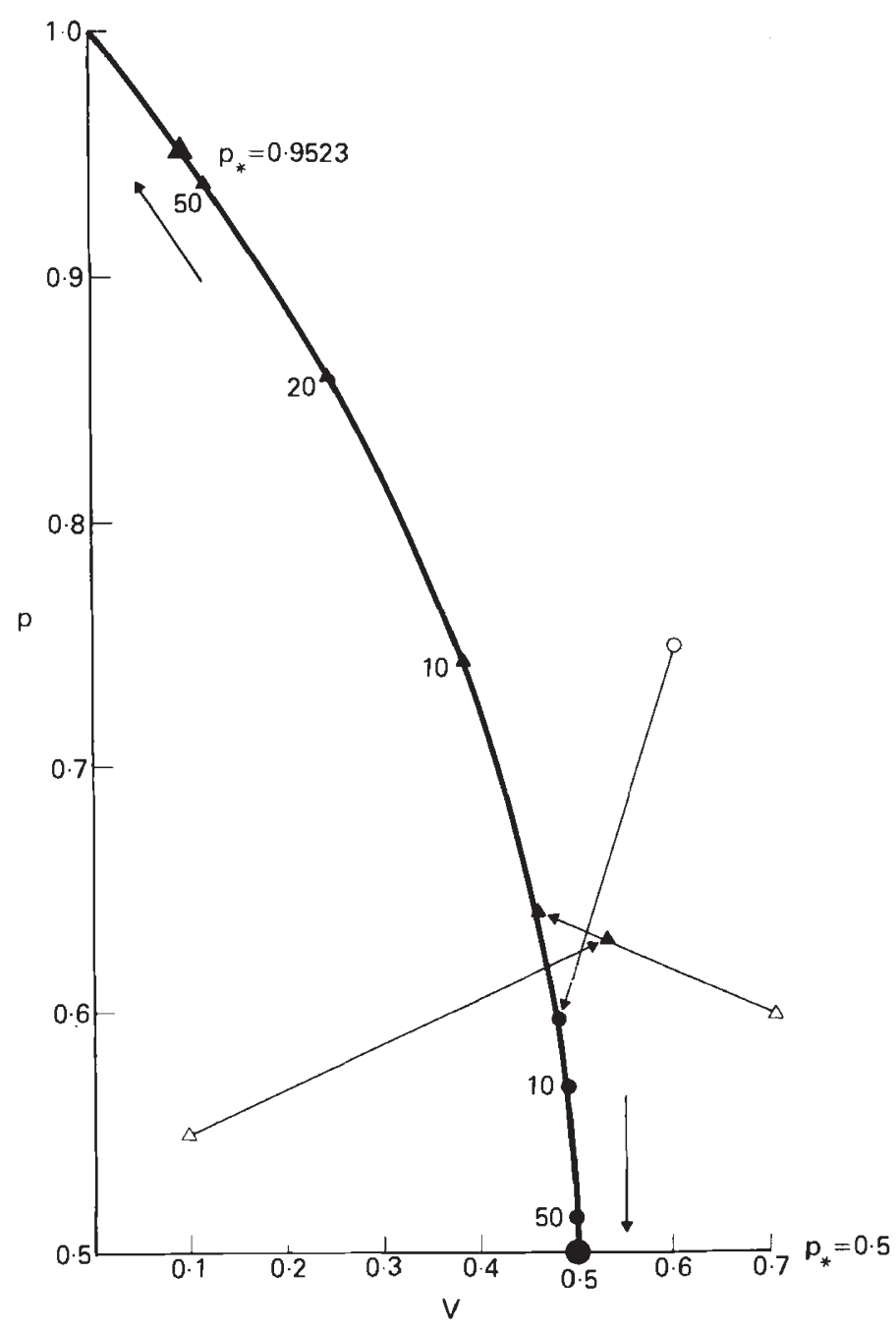

FIG. 1.-Rates of approach to equilibria along the Hardy-Weinberg line. The figure shows the Hardy-Weinberg relationship between gene frequency and heterozygote frequency. Solid triangles indicate successive points in the approach to the equilibrium $p_{*}=0.9523$. Solid circles indicate points in the approach to $p_{*}=0 \cdot 5$. The initial frequencies are indicated by open triangles and circles. After the first two generations, the number of generations is stated beside each point. 
approach to two different equilibria in the encounter model. One is the equilibrium

$$
p_{*}=v_{*}=\frac{1}{2}
$$

and the other is

$$
p_{*}=0.9523 \quad v_{*}=0.0909 .
$$

From various initial points on different sides of the Hardy-Weinberg line, the frequencies in one or two generation become aligned and then follow the line to the equilibria.

\section{CONClusion}

When the heterozygotes are the sexually favoured genotypes, several polymorphic states and the fixation states may all be stable: evolution may produce several alternative outcomes. The process of sexual selection may be described in terms of a general function, $f(v)$, of the heterozygote frequency, $v$. This function gives the relative mating frequency of heterozygotes. There are two equilibrium conditions. There always exists a central equilibrium

$$
p_{*}=v_{*}=\frac{1}{2}
$$

which is stable provided that $f\left(\frac{1}{2}\right)>\frac{1}{2}$. In this case, at equilibrium, the heterozygotes retain their advantage since they continue to mate at higher frequency than their presence in the population. Other equilibria are given by the equation

$$
v_{*}=f\left(v_{*}\right)
$$

There may be several solutions of this equation depending on the function $f$ which describes the mating process. Specific models of mate selection give rise to explicit expressions for $f$ and hence solutions to the equilibrium values. If one solution is given by

$$
v_{*}=0
$$

then either one homozygote or the other becomes fixed in the population. Depending on parameter values, these fixation states may be stable. Other solutions may correspond to stable polymorphic equilibria on one or other side of the central equilibrium.

These results can thus be used to make general qualitative inferences about the outcome of sexual selection in specific models of mate selection. Population cage experiments replicated with different initial frequencies will provide data by which the models can be refuted. If experiments showed that heterozygotes always mated in excess of their frequency so that $f(v)>v$, then the central equilibrium point $p_{*}=v_{*}=\frac{1}{2}$ should be globally stable. Population cages started at different frequencies should all reach the central equilibrium.

A remarkable example of sexual selection in favour of heterozygotes has been reported by Smith (1981). In the butterfly Danaus chrysippus, the form dorippus is semi-dominant to aegyptius. Heterozygotes, known as transiens, closely resemble dorippus. Transiens always mates at considerably higher frequency than its occurrence in populations $(f(v)>v)$. If the 
homozygotes were equal in their mating propensities, the populations of Danaus chrysippus should be stable at the central point of equilibrium. But although the homozygotes are always at a sexual disadvantage compared to heterozygotes, they differ between themselves in their mating frequencies. In the wet and cool season aegyptius has a considerable advantage over dorippus homozygotes and rapidly increases in frequency. But in the hot and dry season, the relative fitnesses of the homozygotes are reversed and dorippus increases in frequency. These changes in the sexual selection of the homozygotes will necessarily give rise to cyclic variation in frequency round the central equilibrium.

\section{REFERENCES}

ClARKE, B., AND O'DONAld, P. 1964. Frequency-dependent selection. Heredity, 19, 201 206.

KARLIN, S. 1978. Comparisons of positive assortative mating and sexual selection models. Theor. Pop. Biol., 14, 281-312.

O'DONALD, P. 1978. Theoretical aspects of sexual selection: a generalized model of mating behavior. Theor. Pop. Biol., 13, 226-243.

O'DONALD, P. 1979. Theoretical aspects of sexual selection: variation in threshold of female mating response. Theor. Pop. Biol., 15, 191-204.

O'DONALd, P. 1980a. Genetic Models of Sexual Selection. Cambridge University Press.

O'DONALD, P. $1980 \mathrm{~b}$. A general analysis of genetic models with frequency-dependent mating. Heredity, 44, 309-320.

SMITH, S. A. S. 1981. Heterozygous advantage expressed through sexual selection in a polymorphic African butterfly. Nature, in press. 